\title{
Quiste broncogénico esofágico: una causa poco común de disfagia en adultos. Reporte de caso y revisión de la literatura
}

\author{
Esophageal bronchogenic cyst: an uncommon cause of dysphagia in adults. Case report \\ and literature review
}

\author{
Ana P. Ceniceros-Cabrales* y Patricio Sánchez-Fernández \\ Servicio de Gastrocirugía, Hospital de Especialidades, Centro Médico Nacional Siglo XXI, Instituto Mexicano del Seguro Social, Ciudad de \\ México, México
}

\section{Resumen}

Antecedentes: Los quistes broncogénicos son lesiones congénitas raras que resultan de brotes anormales del tubo traqueobronquial primitivo. La localización del quiste depende de la etapa embriológica del brote anormal. Aunque los quistes broncogénicos periesofágicos han sido frecuentemente reportados, un quiste completamente intramural es muy raro. Caso clínico: Mujer de 42 años con cuadro de 3 meses de evolución, con dolor retroesternal asociado a la ingestión de alimentos, acompañado de disfagia intermitente a sólidos. Se realiza esofagograma, tomografía de tórax de alta resolución y ultrasonido endoscópico, que concluyen probable quiste broncogénico esofágico. Se realiza resección por cirugía torácica videoasistida, sin complicaciones. La paciente cursa con adecuada evolución y remisión completa de la sintomatología. Conclusión: Los quistes broncogénicos de la pared esofágica son lesiones extremadamente poco comunes. Está indicado su tratamiento quirúrgico al ser sintomáticos; es de elección la resección por cirugía torácica videoasistida, con excelentes resultados a largo plazo y mínima morbilidad.

PALABRAS CLAVE: Quiste broncogénico. Esófago. Disfagia. Cirugía torácica videoasistida.

\begin{abstract}
Backgronund: Bronchogenic cysts result from abnormal budding of the primitive tracheobronchial tube and are rare congenital cystic lesions. The location of the cyst depends on the embryological stage of abnormal budding. Although periesophageal bronchogenic cysts have been frequently reported, a completely intramural cyst is very rare. Clinical case: $A$ 42-year-old female patient, a three-month course with retrosternal pain associated with food intake, accompanied by intermittent dysphagia to solids. Esophagogram, high resolution thoracic tomography and endoscopic ultrasound are performed, concluding a probable esophageal bronchogenic cyst. Resection is performed by video-assisted thoracic surgery, without complications. Patient presents with adequate evolution and complete remission of the symptomatology. Conclusion: Bronchogenic cysts of the esophageal wall are extremely uncommon lesions. Its surgical treatment is indicated to be symptomatic; video-assisted thoracoscopic surgery resection is of choice, with excellent long-term results and minimal morbidity.
\end{abstract}

KEY WORDS: Bronchogenic cyst. Esophagus. Dysphagia. Video-assisted thoracic surgery.

\footnotetext{
Correspondencia:

*Ana Paula Ceniceros-Cabrales

Av. Cuauhtémoc, 330

C.P. 06725, Ciudad de México, México

E-mail: anapaucc@ hotmail.com
}

Fecha de recepción: 20-07-2017

Fecha de aceptación: 27-03-2018

DOI: $10.24875 /$ CIRU.M18000018
Cir Cir. 2018;86:187-190

Contents available at PubMed www.cirugiaycirujanos.com 


\section{Antecedentes}

Los quistes broncogénicos son lesiones congénitas raras que resultan de brotes anormales del tubo traqueobronquial primitivo'. La localización del quiste depende de la etapa embriológica del brote anormal ${ }^{2}$.

Aproximadamente el $36-90 \%$ de los quistes broncogénicos ocurren en el mediastino, y la mayoría de los quistes restantes implican el parénquima pulmonar ${ }^{3}$. Los quistes broncogénicos esofágicos son raros; los paraesofágicos son los más típicamente reportados, y un quiste completamente intramural es muy raro. Los quistes broncogénicos esofágicos intramurales solo se han descrito en 23 casos de adultos desde $1981^{4}$.

La mayoría de los pacientes son recién nacidos o lactantes en el momento de la presentación. Los síntomas son usualmente causados por la compresión de estructuras intratorácicas, con problemas respiratorios predominantes. Los síntomas primarios consisten en dolor retroesternal, tos, disnea o fiebre ${ }^{5}$.

En cuanto al tratamiento de los quistes broncogénicos esofágicos, se recomienda la extirpación quirúrgica completa mediante toracotomía o cirugía torácica videoasistida. Algunos autores lo recomiendan incluso cuando son asintomáticos, por complicaciones posteriores como infección, rotura, hemorragia intraquística o cambios carcinomatosos ${ }^{6}$.

\section{Objetivo}

Describir un caso raro de un quiste broncogénico de la pared esofágica diagnosticado en la edad adulta, el cual se trató mediante resección por cirugía torácica videoasistida, y realizar una revisión de la literatura.

\section{Caso clínico}

Mujer de 42 años, niega enfermedades crónicas-degenerativas, con antecedentes quirúrgicos de dos cesáreas y oclusión tubaria bilateral; resto de antecedentes negados. Inicia su padecimiento actual 3 meses previos a su ingreso, con dolor retroesternal asociado a la ingestión de alimentos, acompañado de disfagia intermitente a sólidos.

Como parte de su protocolo de estudio se realiza esofagograma, tomografía torácica de alta resolución y ultrasonido endoscópico. El esofagograma muestra compresión extrínseca en el tercio medio del esófago (Fig. 1). En la tomografía de tórax se documenta tumoración dependiente del tercio medio del esófago, que rechaza la endoscópico que reporta lesión tipo quística entre los 26 y $31 \mathrm{~cm}$ de la arcada dentaria, la cual mide $39 \times 30 \mathrm{~mm}$ de diámetro, de contenido anecoico, discretamente turbio, que se origina de la pared del esófago y crece hacia dentro cubierta por mucosa esofágica normal y hacia fuera rechazando la tráquea y la aorta, y adenopatía en región subcarinal, con diagnóstico de probable quiste broncogénico vs. quiste de duplicación (Fig. 3).

Se decide realizar resección por cirugía torácica videoasistida, encontrando como hallazgo una lesión de $30 \times 30 \mathrm{~mm}$ en la cara lateral izquierda del esófago, en el tercio medio (Fig. 4), con reporte histopatológico de quiste broncogénico (Fig. 5). La paciente presenta una adecuada evolución posquirúrgica, con incidencia de neumotórax posterior al retiro de la sonda endopleural, que se recolocó y luego se retiró con éxito; es egresada a su domicilio en adecuadas condiciones y con remisión completa de la sintomatología.

\section{Discusión}

Los quistes broncogénicos son anomalías broncopulmonares del intestino anterior que suelen ocurrir a

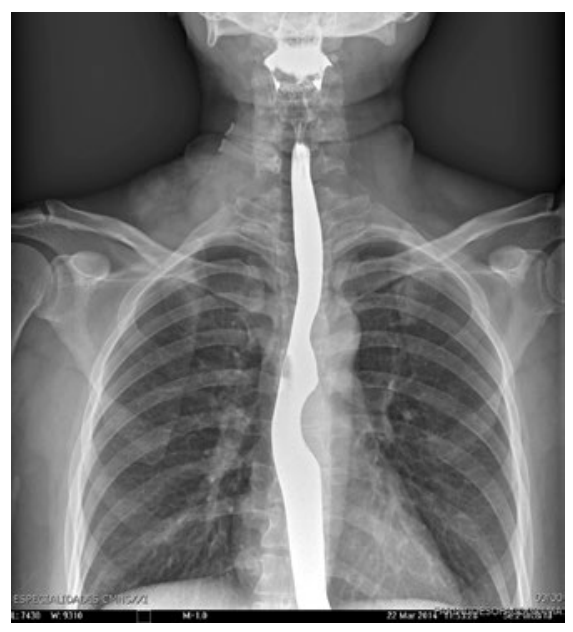

Figura 1. Esofagograma que muestra compresión extrínseca en el tercio medio del esófago.

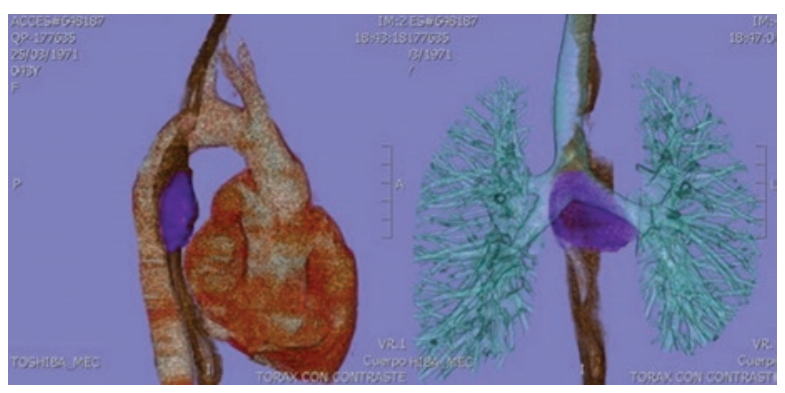

Figura 2. Reconstrucción de tomografía de alta resolución de tórax en la que se observa una tumoración en el tercio medio del esófago, que rechaza la tráquea y la aorta. 


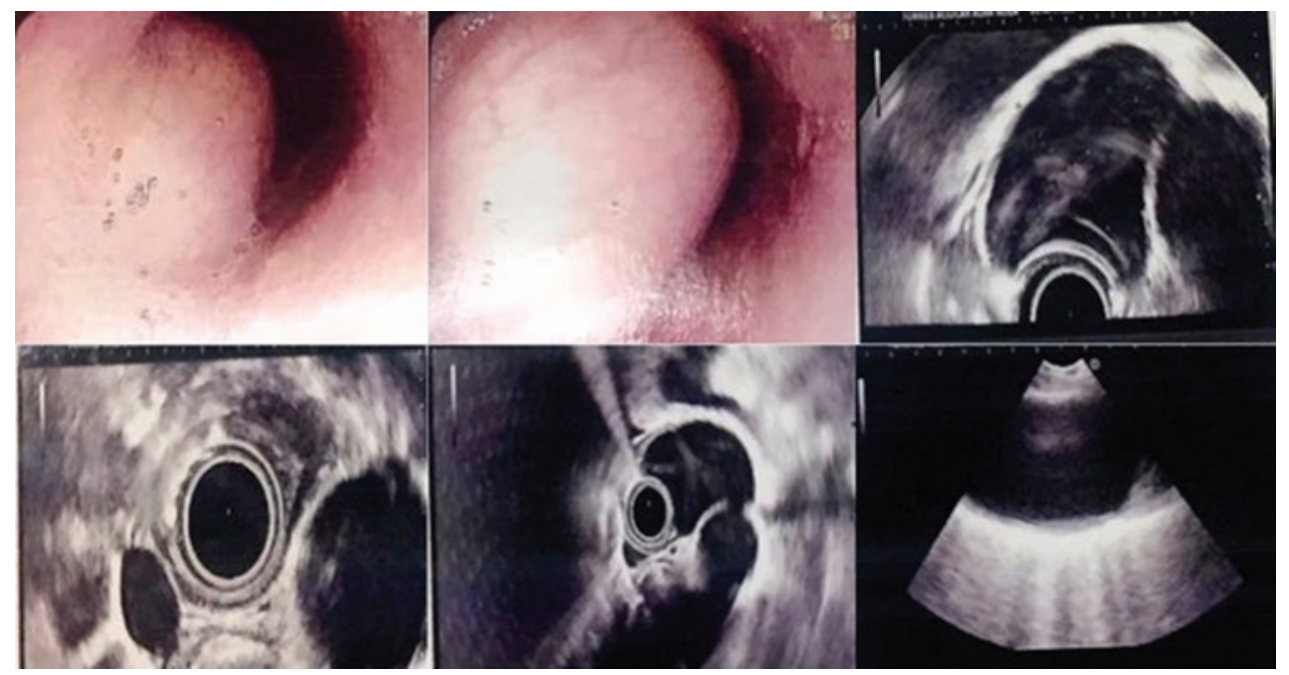

Figura 3. Ultrasonido endoscópico que reportó lesión tipo quística entre los 26 y $31 \mathrm{~cm}$ de la arcada dentaria, de 39 × 30 mm de diámetro, de contenido anecoico, que se origina de la pared del esófago y crece hacia dentro cubierta por mucosa esofágica normal y hacia fuera rechazando la tráquea y la aorta.

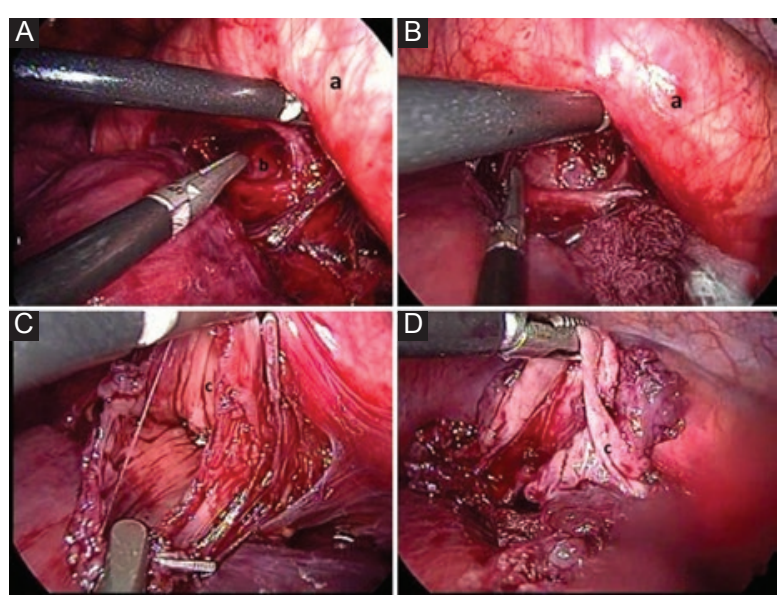

Figura 4. A y B: disección del quiste broncogénico de las estructuras adyacentes. $\boldsymbol{C}$ : apertura del quiste, previa aspiración de su contenido. D: se muestra la pared del quiste ya disecado. a: aorta torácica; b: quiste broncogénico; c: pared del quiste broncogénico.

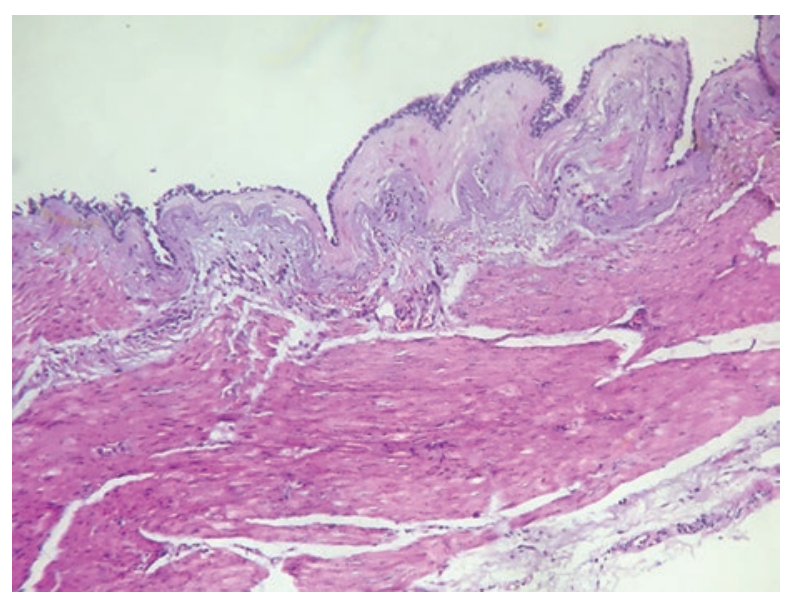

Figura 5. Imagen histológica que muestra el epitelio respiratorio que recubría el interior del quiste (tinción con eosina y hematoxilina, amplificación a 40x). lo largo del árbol traqueobronquial en el mediastino². Pueden estar localizados en la región paratraqueal, carinal o hiliar. Los quistes broncogénicos esofágicos se desarrollan cuando el brote anormal se produce en la ranura laringotraqueal después de la tercera semana de gestación ${ }^{2,7}$.

Histológicamente, los quistes broncogénicos se caracterizan por la presencia de epitelio cilíndrico ciliado y diversos componentes de músculo liso, glándula bronquial, cartílago e incluso alvéolos pulmonares en la pared del quiste, dependiendo de la etapa de la gestación en que se produce el brote anómalo².

Cuando están presentes, los síntomas suelen ser causados por la compresión de las estructuras circundantes o por complicaciones relacionadas con el quiste $^{8}$. Las complicaciones más comunes son hemorragia intraquística, infección, inflamación, ulceración del quiste, y rotura o perforación del quiste en el árbol traqueobronquial, pericardio o pleura. Una masa quística mediastínica de 3-4 cm adyacente al esófago que se presenta con disfagia y dolor torácico es altamente sugerente de quiste broncogénico².

Diferentes técnicas de imagen ayudan a localizar la anomalía y a detectar cualquier lesión asociada. La radiografía de tórax posteroanterior y lateral, que es la modalidad diagnóstica primaria, puede demostrar la localización del quiste, su tamaño y contornos, y especialmente puede mostrar niveles de aire-líquido en el quiste. El esofagograma con contraste puede demostrar si existe una comunicación entre el quiste y el esófago, o revelar solo una compresión lisa extrínseca y desplazamiento del esófago. La 
tomografía computarizada es útil para evaluar la naturaleza quística de la masa, mostrando una densidad homogénea casi de agua; puede indicar la ubicación exacta de la masa y sus relaciones con otras estructuras torácicas ${ }^{9}$. Sin embargo, si el quiste está infectado o tiene un alto contenido de proteínas y calcio, su densidad puede caer dentro del rango de tejidos sólidos, lo que aumenta la incertidumbre diagnóstica ${ }^{10}$. El diagnóstico definitivo se realiza después de la extirpación quirúrgica con confirmación histológica11.

La resonancia magnética es sensible para detectar tejido lleno de líquido. Sin embargo, muchos quistes broncogénicos contienen grandes cantidades de material proteínico, produciendo así imágenes hiperintensas en T1, lo que dificulta la diferenciación de las lesiones grasas. La endoscopia se realiza para descartar la presencia de malignidad ${ }^{10}$. El ultrasonido endoscópico es útil para diagnosticar quistes broncogénicos esofágicos intramurales, mediante una delimitación clara de la localización submucosa del quiste ${ }^{6}$.

El tratamiento de los quistes broncogénicos sigue siendo controvertido. Si el quiste es sintomático, puede realizarse enucleación convencional o toracoscópica. El propósito de la cirugía es el diagnóstico, la resección y la prevención de complicaciones. La cirugía toracoscópica videoasistida es una opción menos invasiva, pero con una tasa de complicaciones del $10-30 \%^{4,12}$. Las alternativas a la cirugía incluyen la aspiración por aguja transesofágica, e incluso se ha descrito una extirpación exitosa por resección endoscópica transoral para un quiste localizado en la pared esofágica ${ }^{13-15}$. La resección quirúrgica radical del quiste broncogénico, independientemente del método quirúrgico, ofrece resultados satisfactorios, sin recurrencia a largo plazo ${ }^{2}$.

\section{Conclusión}

Los quistes broncogénicos de la pared esofágica son lesiones extremadamente poco comunes. Está indicado su tratamiento quirúrgico al ser sintomáticos, y es de elección la resección por cirugía torácica videoasistida, con excelentes resultados a largo plazo y mínima morbilidad.

\section{Responsabilidades éticas}

Protección de personas y animales. Los autores declaran que los procedimientos seguidos se conformaron a las normas éticas del comité de experimentación humana responsable y de acuerdo con la Asociación Médica Mundial y la Declaración de Helsinki.

Confidencialidad de los datos. Los autores declaran que han seguido los protocolos de su centro de trabajo sobre la publicación de datos de pacientes.

Derecho a la privacidad y consentimiento informado. Los autores han obtenido el consentimiento informado de los pacientes y/o sujetos referidos en el artículo. Este documento obra en poder del autor de correspondencia.

\section{Conflicto de intereses}

Los autores declaran no tener ningún conflicto de intereses.

\section{Financiamiento}

Los autores declaran no haber recibido financiación para la realización de este trabajo.

\section{Bibliografía}

1. St-Georges R, Deslauriers J, Duranceau A, Vaillancourt R, Deschamps $\mathrm{C}$, Beauchamp $\mathrm{G}$, et al. Clinical spectrum of bronchogenic cysts of the mediastinum and lung in the adult. Ann Thorac Surg. 1991;52:6-13.

2. Ko S, Hsieh M, Lin J, Huang C, Li C, Cheung Y, et al. Bronchogenic cyst of the esophagus: clinical and imaging features of seven cases. Clin Imag. 2006; 30:309-14.

3. Berrocal T, Madrid C, Novo S, Gutiérrez J, Arjonilla A, Gómez-León N. Congenital anomalies of the tracheobronchial tree, lung, and mediastinum: embryology, radiology, and pathology. Radiographics. 2004;24:27.

4. Turkyilmaz A, Eroglu A, Subasi M, Findik G. Intramural esophageal bronchogenic cysts: a review of the literature. Dis Esophagus. 2007;20:461-5.

5. Nobuhara K, Gorski Y, Quaglia M, Shamberger R. Bronchogenic cysts and esophageal duplications: common origins and treatment. J Pediatr Surg. 1997;37:1408-13.

6. Han C, Lin R, Yu J, Zhang Q, Zhang Y, Liu J, et al. A case report of esophageal bronchogenic cyst and review of the literature with an emphasis on endoscopic ultrasonography appearance. Medicine. 2016; 95:11.

7. Westerterp M, Van den Berg, J, Van Lanschot J, Fockens P. Intramural bronchogenic cysts mimicking solid tumors. Endoscopy. 2004; 36:1119-22

8. Altieri M, Zheng R, Pryor A, Heimann A, Ahn S, Telem D. Esophageal bronchogenic cyst and review of the literature. Surg Endosc. 2015; 29:3010-5.

9. Yang X, Partanen K, Seppa A, Berg E, Pasanen P. Paraesophageal bonchogenic cyst in adult: can it be differentiated from intramural esophageal cyst by different imagenings? Clinical Imagin. 1994;18:68-71.

10. Lim L, Ho K Goh P. Preoperative diagnosis of a paraesophageal bronchogenic cyst using endosonography. Ann Thorac Surg. 2002;73:633-5.

11. Chuang K, Huang T, Cheng Y, Chen J, Tzao C, Chang H, et al. Esophageal bronchogenic cyst: a rare entity. Z Gastroenterol. 2007; 45:958-60.

12. Chafik A, Benjelloun A, Qassif H, El Fikri A, El Barni R, Zrara I. Intramural esophageal bronchogenic cysts. Asian Cardiovascular \& Thoracic Annals. 2011;19:69-71.

13. Watson DI, Britten-Jones R. Thoracoscopic excision of bronchogenic cyst of the esophagus. Surg Endosc. 1995;9:824-5.

14. Sashiyama H, Miyazaki S, Okazaki Y, Kaiho T, Nakajima Y, Hoshino $T$, et al. Esophageal bronchogenic cyst successfully excised by endoscopic mucosal resection. Gastrointest Endosc. 2002;56:141-5.

15. Martinod E, Pons F, Azorín J, Mouroux J, Dahan M, Faillon JM, et al. Thoracoscopic excision of mediastinal bronchogenic cysts: results in 20 cases. Ann Thorac Surg. 2000;69:1525-8. 